\title{
Flow Control of Duct System
}

\author{
By Yasushi Niitsu and Meiji Kurahashi \\ Faculty of Engineering, Osaka University
}

\begin{abstract}
The following article contains the results of experiments by the authors with two methods of obtaining uniform duct-system distribution of air flow from each branch pipe or of adjusting air flow to specific conditionswithout changing the existing duct system.

Method No. 1 starts with the fundamental flow equation and proceeds by stages to finding out unknown factors to be controlled.

Method No. 2, which cannot be described in the scope of this preface, has been shown by the experiments to be simpler and more practical.

As to the duct system with parallel blowers, the authors have reached the conclusion that uniform flow from each branch pipe can be obtained more easily by adjusting the revolutions of blowers than by inserting the control resistance body.
\end{abstract}

\section{Introduction}

To obtain a uniform distributicn of temperature and humidity in spinning mills, it is most essential that air flow from each branch pipe in the duct system be uniform. But in any given ducts of the conventional design, the flow is not always distributed uniformly.

We have made a series of practical studies on air flow from the duct system and have published $[1] \sim[4]$ our findings on previous occasions. In the present article, we shall confine ourselves to a discussion of methods of obtaining uniform distribution of air flow or adjusting it to desired conditions by means of the existing duct system without changing its form.

\section{Fundamental Flow Equation by Duct System}

Before proceeding to our discussion, we list the nomenclatures used in this article:

$Q, q$ : flow quantity of duct in main pipe or branch pipe $\mathrm{kg} / \mathrm{s}$

$W, w$ : velocity of duct in main pipe or branch pipe $\mathrm{m} / \mathrm{s}$

$P, P_{0}$ : pressure in duct section or outside of pipe line $\mathrm{kg} / \mathrm{m}^{2}$

$L, l$ : length of duct in main pipe or branch pipe $\mathrm{m}$

$D, d$ : diameter of duct in main pipe or branch pipe $\mathrm{m}$

$F, f$ : area of duct section in main pipe or branch pipe $\mathrm{m}^{2}$

$\zeta_{d} \quad$ : resistance coefficient of separate flow or confluent flow from main pipe to main pipe

$\zeta_{\delta} \quad$ : resistance coefficient of separate flow or confluent flow from main pipe to branch pipe $\zeta \quad:$ resistance coefficient

$K, K^{\prime}:$ synthetic resistance coefficient of mian pipe

$k$ : synthetic resistance coefficient of branch pipe

$k^{\prime}, k^{\prime \prime}$ : resistance coefficient of inserted resistance bcdy to change the flow condition

$\lambda \quad$ : coefficient of fluid friction

$\gamma$ : specific weight $\mathrm{kg} / \mathrm{m}^{3}$

$r$ : ratio of flow quantity-with the suffixes $i$ meaning inlet; 0 , outlet; $s$, suction or exhaust of pipe of blower.

Sufixes $1,2, \cdots \cdots, n$ represent the main or branch pipes number and show the flow condition.

(A) In the Case of Blow Duct

In the typical duct system with branch pipe numbered $n$ as shown in Figure 1 , the flow from main pipe of blower to branch pipe $n$ is, generally, as follows:

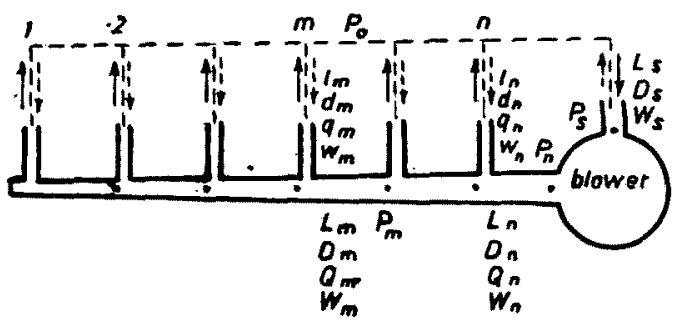

Fig. 1

$$
\begin{aligned}
P_{n} & +\frac{\gamma}{2 g} W_{n}^{2}=\lambda \frac{L_{n}}{D_{n}} \frac{\gamma}{2 g} W_{n}^{2}+\zeta b n \frac{\gamma}{2 g} W_{n}^{2} \\
& +\lambda \frac{l_{n}}{d_{n}} \frac{\gamma}{2 g} W_{n}^{2}+\zeta_{o n} \frac{\gamma}{2 g} W_{n}^{2}+\frac{\gamma}{2 g} W_{x}^{2}+P_{0} \\
& \cdots \cdots \cdots \cdots \cdots \cdots \cdots \cdots(1)
\end{aligned}
$$




$$
P_{n}-P_{s}=\left\{\lambda \frac{L_{n}}{D_{n}}+\zeta_{b n}+\left(1+\lambda \frac{L_{s}}{D_{s}}+\zeta_{s}\right)\left(\frac{W_{s}}{W_{n}}\right)^{2}-1\right\} \frac{\gamma}{2 g} W_{n}^{2}+\left(\lambda \frac{l_{n}}{d_{n}}+\zeta_{o n}+1\right) \frac{\gamma}{2 g} W_{n}^{2}
$$

However, from the equation of continuous flow, we have

$$
\begin{gathered}
W_{n} F_{n} \gamma=Q_{n}, w_{n} f_{n \gamma}=q_{n} \ldots \ldots \ldots \ldots \ldots \ldots \ldots \ldots \ldots \ldots \ldots \ldots \ldots \ldots \ldots \\
W_{s} F_{s} \gamma=Q_{n} \quad \therefore\left(\frac{W_{s}}{W_{n}}\right)^{2}=\left(\frac{F_{n}}{F_{s}}\right)^{2} \\
\left\{\lambda \frac{L_{n}}{D_{n}}+\zeta_{s n}+\left(1+\lambda \frac{L_{s}}{D_{s}}+\zeta_{s}\right)\left(\frac{F_{n}}{F_{s}}\right)^{2}-1\right\} \frac{1}{2 g \gamma}\left(\frac{1}{F_{n}}\right)^{2}=K_{n} \\
\left(\lambda l_{n}+\zeta_{n n}+1\right) \frac{1}{2 g \gamma}\left(\frac{1}{f_{n}}\right)^{2}=k_{n}
\end{gathered}
$$

$\therefore P_{n}-P_{s}=K_{n} Q_{n}^{2}+k_{n} q_{n}^{2}$

With the flow of blow $b(n-1)$ blower, we have

$$
\begin{aligned}
& P_{n}+\frac{\gamma}{2 g} W_{n}^{2}=\lambda \frac{L_{n}}{D_{n}} \frac{\gamma}{2 g} W_{n}^{2}+\zeta_{\ln } \frac{\gamma}{2 g} W_{n}^{\prime}+\lambda \frac{L_{(n-1)}}{D_{(n-1)}} \frac{\gamma}{2 g} W_{(n-1)}^{2}+\zeta_{b(n-1)} \frac{\gamma}{2 g} W_{(n-1)}^{2}+\lambda \frac{l_{(n-1)}}{d_{(n-1)}} \frac{\gamma}{2 g} w_{(n-1)}^{2} \\
& +\frac{\gamma}{2 g} w_{(n-1)}^{2}+\zeta_{o(n-1)} \frac{\gamma}{2 g} w_{(n-1)}^{2}+\zeta_{s} \frac{\gamma}{2 g} W_{n}^{2}+\lambda \frac{L_{s}}{D_{s}} \frac{\gamma}{2 g} W_{s}^{2}+P_{s}+\frac{\gamma}{2 g} W_{s}^{2} \\
& \therefore P_{n}-P_{s}=\left\{\lambda \frac{L_{n}}{D_{n}}+\zeta_{d n}+\left(1+\lambda \frac{L_{s}}{D_{s}}+\zeta_{s}\right)\left(\frac{F_{n}}{F_{s}}\right)^{2}-1\right\} \frac{\gamma}{2 g} W_{x}^{2}+\left(\lambda \frac{L_{(n-1)}}{D_{(n-1)}}+\zeta_{b(n-1)}\right) \frac{\gamma}{2 g} W_{(n-1)}^{2} \\
& +\left(\lambda \frac{l_{(n-1)}}{d_{(n-1)}}+\zeta_{0(n-1)}+1\right) \frac{\gamma}{2 g} w_{(n-1)}^{2} \\
& \left\{\lambda \frac{L_{n}}{D_{n}}+\zeta_{d n}+\left(1+\lambda \frac{L_{s}}{D_{s}}+\zeta_{s}\right)\left(\frac{F_{n}}{F_{s}}\right)^{2}-1\right\} \frac{1}{2 g \gamma}\left(\frac{1}{F_{n}}\right)^{2}=K_{n}^{\prime} \\
& \left(\lambda \frac{L_{(n-1)}}{D_{(n-1)}}+\zeta_{b(n-1)}\right) \frac{1}{2} g \gamma\left(\frac{1}{F_{(n-1)}}\right)^{2}=K_{(n-1)} \\
& \left(\lambda \frac{l_{(n-1)}}{d_{(n-1)}}+\zeta_{0(n-1)}+1\right) \frac{1}{2 g}\left(\frac{1}{f_{(n-1)}}\right)^{2}=k_{(n-1)} \\
& \therefore P_{n}-P_{s}=K_{n}^{0} Q_{n}^{a}+K_{(n-1)} Q_{(n-1)}^{a}+k_{(n-1)} q_{(n-1)}^{a}
\end{aligned}
$$

Accordingly, if the relation $\left(P_{n}-P_{8}\right)$ is equal to the blower pressure $P$, general flow equa tion may be expressed as following:

$$
P=\stackrel{n}{m+1}_{\sum_{m}^{\prime}} K_{m}^{\prime} Q_{m}^{2}+K_{m} Q_{m}^{2}+k_{m} q_{m}^{2}
$$

where

$$
\begin{aligned}
& K_{m}=\left(\lambda \frac{L_{m}}{D_{m}}+\zeta_{3 n z}\right) \frac{1}{2 g \gamma}\left(\frac{1}{F_{m}}\right)^{2} \\
& K_{m}^{\prime}=\left(\lambda \frac{L_{m}}{D_{m}}+\zeta_{d m}\right) \frac{1}{2 g \gamma}\left(\frac{1}{F_{m}}\right)^{2} m<n \\
& k_{m}=\left(\lambda \frac{l_{m}}{d_{m}}+\zeta_{o m}+1\right) \frac{1}{2 g \gamma}\left(\frac{1}{f_{m}}\right)^{2}
\end{aligned}
$$

(B) In the Case of Suction Duct.

Following the same manner as (A), we have the following relative equations:

$$
\begin{aligned}
P_{n}+ & \frac{\gamma}{2 g} W_{n}^{2}=P_{\theta}-\lambda \frac{L_{n}}{D_{n}} \frac{\gamma}{2 g} W_{n}^{2}-\zeta_{n n} \frac{\gamma}{2 g} W_{n}^{2} \\
& \quad-\lambda \frac{l_{n}}{d_{n}} \frac{\gamma}{2 g} w_{n}^{2}-\zeta_{i n} \frac{\gamma}{2 g} w_{n}^{2} \ldots \ldots \ldots \ldots \ldots(1) \\
P_{s}+ & \frac{\gamma}{2 g} W_{s}^{2}=\zeta_{s} \frac{\gamma}{2 g} W_{s}^{2}+\lambda \frac{L_{s}}{D_{s}} \frac{\gamma}{2 g} W_{s}^{2} \\
& +P_{0}+\frac{\gamma}{2 g} W_{s}^{2} \ldots \ldots \ldots \ldots \ldots \ldots \ldots \ldots(1)
\end{aligned}
$$

and also

$$
\begin{aligned}
\therefore P_{s} & -P_{n}=\left\{\lambda \frac{L_{n}}{D_{n}}+\zeta_{i n}+\left(\lambda \frac{L_{s}}{D_{s}}+\zeta_{s}\right)\left(\frac{F_{n}}{F_{s}}\right)^{2}\right. \\
+1 & \} \frac{\gamma}{2 g} W_{n}^{2}+\left(\lambda \frac{l_{n}}{d_{n}}+\zeta_{i n}\right) \frac{\gamma}{2 g} w_{n}^{2} \cdots
\end{aligned}
$$

$$
\begin{aligned}
P_{s}- & P_{n}=\left\{\lambda \frac{L_{n}}{D_{n}}+\zeta_{d n}+\left(\lambda \frac{L_{s}}{D_{s}}+\zeta_{s}\right)\left(\frac{F_{n}}{F_{s}}\right)^{2}\right. \\
& +1\} \frac{\gamma}{2 g} W_{n}^{2}+\left\{\lambda \frac{L_{(n-1)}}{D_{(n-1)}}+\zeta_{h(n-1)}\right\} \frac{\gamma}{2 g} W_{(n-1)}^{2} \\
& +\left(\lambda \frac{l_{(n-1)}}{d_{(n-1)}}+\zeta_{i(n-1)}\right) \frac{\gamma}{2 g} w_{(n-1)}^{9} \quad \cdots \cdots(14) \\
K_{m}= & \left(\lambda \frac{L_{m}}{D_{m}}+\zeta_{i m}\right) \frac{1}{2 g \gamma}\left(\frac{1}{F_{m}}\right)^{2} \\
K_{m}= & \left.\left(\lambda \frac{L_{m}}{D_{m}}+\zeta_{d m}\right) \frac{1}{2 g \gamma}\left(\frac{1}{F_{m}}\right)^{2}, m<n\right\} \cdots(15) \\
k_{m}= & \left(\lambda \frac{l_{m}}{d_{m}}+\zeta_{i n}\right) \frac{1}{2 g \gamma}\left(\frac{1}{f_{m}}\right)^{2}
\end{aligned}
$$

By using (15), the equations of (13), (14) are obtainable in the same form as equation (10).

2. Uniform Distribution of Branch Pipe Flow (which we will call Method No.1)

In any given duct system having unknown 
pressure loss coefficient, we assume that the following are already known:

(1) Flow rate of branch pipe, that is, $K_{m}$, $K_{n s}^{\prime}$ are unknown and $k_{m}, q_{m}$ known.

(2) Performance of blower is known and the relation between $P$ and $Q_{n}$ is also known. And when each branch pipe flow is uniformly distributed, resistance values $\Delta k$ to be inserted are as follows:

$$
\begin{aligned}
q_{1}= & q_{2}=\cdots \cdots=q_{(n-1)}=q \equiv q \cdots \cdots \cdots \\
P= & K_{n} Q_{n}^{2}+k_{n} q^{2}+\Delta k_{n} q^{2} \\
= & K_{n}^{\prime} Q_{n}^{2}+K_{(n-1)} Q_{(n-1)}^{2}+k_{(n-1)} q^{2} \\
& +\Delta k_{(n-1)} q^{2}=\cdots \cdots \cdots \cdots \cdots \cdots \\
= & \sum_{m+1}^{n} K_{m}^{\prime} Q_{m}^{2}+K_{m} Q_{m}^{2}+k_{m} q^{2} \\
& +\Delta k_{m} q_{2}=\ldots \ldots \ldots \ldots \cdots \cdots \cdots \\
= & \sum_{2}^{n} K_{m}^{\prime} Q_{m}^{2}+K_{1} Q_{1}^{2}+k_{1} q^{2}+\Delta k_{1} q^{2}
\end{aligned}
$$

And we have following equations for the flow condition 1,2 and 3 .

$$
\begin{aligned}
& P_{1}=\sum_{m+1}^{n} K_{m 1}^{\prime} Q_{m 1}^{2}+K_{m} Q_{m 1}^{2}+k_{m} q_{m 1}^{2}
\end{aligned}
$$

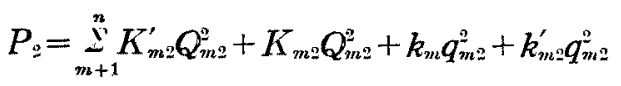

$$
\begin{aligned}
& P_{3}=\sum_{m+1}^{n} K_{m 3}^{\prime} Q_{m 3}^{2}+K_{m 3} Q_{m 3}^{2}+k_{m} q_{m 3}^{2}+k_{m 3}^{\prime \prime} q_{m 3}^{2} \\
& P_{m 1}=K_{m 1} Q_{m 1}^{2}+k_{m} q_{m 1}^{2} \\
& P_{m 2}=K_{m 2} Q_{m 2}^{2}+k_{m} q_{m 2}^{2}+k_{m 2}^{\prime} q_{m 2}^{3} \\
& P_{m 3}=K_{m 3} Q_{m 3}^{2}+k_{m} q_{m 3}^{2}+k_{m 3}^{\prime \prime} q_{m 3}^{2}
\end{aligned}
$$

By expanding $K_{m}$ and $\underset{m+1}{\stackrel{n}{n}} K_{m}^{\prime}$ in the above equation (18) into Taylor series under condition 1 and neglecting terms higher than the second order, the following equation is derived:

$$
\begin{gathered}
K_{m}, \sum_{m+1}^{n} K_{m}^{\prime}=f\left(r_{m 1}\right)+\left(\frac{d f}{d r_{m}}\right)_{r_{m=r_{m 1}}}\left(r_{\mathrm{m}}-r_{m \mathrm{l}}\right) \\
+\frac{1}{2 !}\left(\frac{d^{2} f}{d r_{m}^{2}}\right)_{r_{m=r_{m 1}}}\left(r_{m}-r_{m 1}\right)^{2} \quad \cdots \cdots(20)
\end{gathered}
$$

By using the above equations, $\left(d f / d r_{m}\right) r_{m}=r_{m 1}^{\prime}$, $\left(d^{2} f / d r_{m}^{2}\right) r_{m}=r_{m 1}$ are calculated, so the value of $K_{m}, \sum_{m+1}^{n} K_{m}^{\prime}$ in the uniform distribution is evaluated and controlling resistance value to be inserted by substitution of this value in equation (17) can be arrived at.

However, this methcd requires not only the value of static pressure in branch pipe, but also the value of static pressure in each main pipe section.

3. Uniform Distribution of Branch Pipe Flow (by which we will call Method No. 2)

$$
\begin{aligned}
P= & \sum_{m+1}^{n} K_{m}^{\prime} Q_{m}^{2}+K_{m} Q_{m}^{q}+k_{m} q_{m u}^{2}=\left(K_{m}\right) Q_{n}^{2} \\
& +k_{m} q_{m}^{2}
\end{aligned}
$$

As shown in the above relation, we assume that blower pressuer $P$ consists of two parts: one, branch pipe term with constant factor; the other, total flow term of main pipe with variable factor.

By figuring this relation in terms of the uniform flow condition, we arrive at the following formula:

$$
\begin{aligned}
P & =\left(K_{n}\right) Q_{n}^{2}+\left(k_{n}+\Delta k_{n}\right) q^{2} \\
& =\left(K_{(n-1)}\right) Q_{n}^{2}+\left(k_{(n-1)}+\Delta k_{(n-1)}\right) q_{2}=\cdots \\
& =\left(K_{m}\right) Q_{n}^{2}+\left(k_{m}+\Delta k_{m}\right) q^{2}=\cdots \cdots \cdots \cdots( \\
& =\left(K_{2}\right) Q_{n}^{2}+\left(k_{2}+\Delta k_{2}\right) q^{2} \\
& =\left(K_{1}\right) Q_{n}^{2}+\left(k_{1}+\Delta k_{1}\right) q^{2}
\end{aligned}
$$

If we place $(a)=(b)$ together in the above equation

$$
\begin{gathered}
\left(K_{n}\right) Q_{n}^{2}+\left(k_{n}+\Delta k_{n}\right) q^{2}=\left(K_{(n-1)}\right) Q_{n}^{2} \\
+\left(k_{(n-1)}+\Delta k_{(n-1)}\right) q^{2} \\
\therefore\left\{\left(k_{(n-1)}-k_{n}\right)+\left(\Delta k_{(n-1)}-\Delta k_{n}\right)\right\} \\
=\left\{\left(K_{n}\right)-\left(K_{(n-1)}\right)\right\}\left(\frac{Q_{n}}{q}\right)^{2}
\end{gathered}
$$

and also

$$
Q_{n}=n q \quad \therefore\left(\frac{Q_{n}}{q}\right)^{2}=n^{2}
$$

Accordingly

$$
\begin{aligned}
& \left\{\left(k_{n}-k_{(n-1)}\right)+\left(\Delta k_{n}-\Delta k_{(n-1)}\right)\right\} \\
& \quad=\left\{\left(K_{(n-1)}\right)-\left(K_{n}\right)\right\} n^{2} \\
& \quad\left\{\left(k_{(n-1)}-k_{(n-2)}\right)+\left(\Delta k_{(n-1)}-\Delta k_{(n-2)}\right)\right\} \\
& \quad=\left\{\left(K_{(n-2)}\right)-\left(K_{(n-1)}\right)\left\{n^{2} \cdots \cdots \cdots\right.\right. \\
& \left\{\left(k_{m}-k_{(m-1)}\right)+\left(\Delta k_{n m}-\Delta k_{(m-1)}\right)\right\} \\
& \quad=\left\{\left(K_{(m-1)}\right)-\left(K_{m}\right)\left\{n^{2} \ldots \ldots \ldots \ldots\right.\right. \\
& \left\{\left(k_{2}-k_{1}\right)+\left(\Delta k_{2}-\Delta k_{1}\right)\right\} \\
& \quad=\left\{\left(K_{1}\right)-\left(K_{2}\right)\left\{n^{2}\right.\right.
\end{aligned}
$$

If each branch pipe is the same, then:

$$
k_{1}=k_{2}=\cdots \cdots=k_{m}=\cdots \cdots=k_{(n-1)}=k_{n} \equiv k
$$

Accordingly, resistance coefficient to be inserted is as follows:

$$
\begin{aligned}
& \Delta k_{m}-\Delta k_{(m-1)}=\left\{\left(K_{(m-1)}\right)-\left(K_{m}\right)\left\{n_{2} \cdots(24)\right.\right. \\
& \left(\zeta_{m}\right)=\frac{m}{\perp}\left(\Delta k_{m}-\Delta k_{(m-1)}\right) \cdots \cdots \cdots \cdots \cdots(25) \\
& \Delta P_{m}=\left(\zeta_{m}\right) q^{2}=\zeta_{m} \frac{1}{2 g \gamma}\left(\frac{1}{f_{m}}\right)^{2} q^{2} \ldots \ldots \ldots(26)
\end{aligned}
$$

Also, if we use an orifice or nozzle as the controlling resistance bcdy[5]

$$
\begin{aligned}
& \zeta_{m}=\left(\frac{1}{m^{\prime}}-1\right)\left(\frac{2.75}{m^{\prime}}-1.56\right) \cdots(\text { orifice }) \\
& \zeta_{m}=\left(\begin{array}{c}
1 \\
m^{\prime}
\end{array}-1\right)\left(\frac{1.04}{m^{\prime}}-1\right) \cdots \cdots(\text { nozzle })
\end{aligned}
$$


where:

$$
m^{\prime}=\left(\begin{array}{c}
d \\
D
\end{array}\right)^{2}, \begin{aligned}
& d=\text { diameter of orifice or nozzle. } \\
& D=\text { diameter of branch pipe. }
\end{aligned}
$$

Generally, $\left(K_{n}\right)$ is the function of $r=\left(q_{n} / Q_{m}\right)$. By Taylor expansion based on the present condition $1,\left(K_{m}\right)$ may be expressed thus:

$$
\begin{aligned}
\left(K_{m}\right) & =f\left(r_{1}\right)+\left(\frac{d f}{d r}\right)_{r=r_{1}}\left(r-r_{1}\right) \\
& +\frac{1}{2 !}\left(\frac{d^{2} f}{d r^{2}}\right)_{r=r_{1}}\left(r-r_{1}\right)^{2} \ldots \ldots \ldots . . . \\
& +\frac{1}{3 !}\left(\frac{d^{3} f}{d r^{3}}\right)_{r=r_{1}}\left(r-r_{1}\right)^{3}+\cdots \cdots
\end{aligned}
$$

In the condition of uniform distribution of flow, the value of $r=q_{m} / Q_{m}$ equals $1 / n$, therefore we have:

$$
\begin{aligned}
\left(K_{m}\right)_{r=} \frac{1}{n} & =f\left(r_{1}\right)+\left(\frac{d f}{d r}\right)_{r=r_{1}}\left(\frac{1}{n}-r_{1}\right) \\
& +\frac{1}{2 !}\left(\frac{d^{2} f}{d r^{2}}\right)_{r=r_{1}}\left(\frac{1}{n}-r_{1}\right)^{2} \\
& +\frac{1}{3 !}\left(\frac{d f}{d r^{3}}\right)_{r_{m} r_{1}}\left(\frac{1}{n}-r_{1}\right)^{3}+\ldots \ldots
\end{aligned}
$$

(A) First Approximation

$$
\begin{aligned}
& \left(K_{m}\right)_{r=\frac{1}{n}}=f\left(r_{1}\right)+\left(\frac{d f}{d r}\right)_{r=r_{1}}\left(\frac{1}{n}-r_{1}\right) \\
& P_{2}=\left(K_{m}\right)_{2} Q_{n: 2}^{2}+\left(k_{m_{m}}+k_{m 2}^{\prime}\right) q_{m}^{2} \ldots \ldots \ldots \ldots . \\
& \left(K_{m}\right)_{2}=f\left(r_{1}\right)+\left(\frac{d f}{d r}\right)_{r_{-} r_{1}}\left(r_{2}-r_{1}\right) \\
& \therefore\left(\frac{d f}{d r}\right)_{r_{-} r_{1}}=\frac{\left(K_{m}\right)_{2}-f\left(r_{1}\right)}{\left(r_{2}-r_{1}\right)}
\end{aligned}
$$

With the value of $\left(K_{m}\right)$ known as above, the controlling resistance value can be figured out. (B) Second Approximation

$$
\begin{aligned}
& \left(K_{m}\right)_{r=\frac{1}{n}}=f\left(r_{1}\right)+\left(\frac{d f}{d r}\right)_{r_{-} r_{1}}\left(\frac{1}{n}-r_{1}\right) \\
& \quad+\frac{1}{2 !}\left(\frac{d^{2} f}{d r^{2}}\right)_{r=r_{1}}\left(\frac{1}{n}-r_{1}\right)^{2} \ldots \ldots \ldots \ldots \\
& P_{3}=\left(K_{m}\right)_{3} Q_{n 3}^{2}+\left(k_{m}+k_{n 3}^{\prime \prime}\right) q_{m}^{2} \ldots \ldots \ldots \ldots \\
& \left(K_{m}\right)_{3}=f\left(r_{1}\right)+\left(\frac{d f}{d r}\right)_{r=r_{1}}\left(r_{3}-r_{1}\right) \\
& \quad+\frac{1}{2 !}\left(\frac{d^{2} f}{d r^{2}}\right)_{r=r_{1}}\left(r_{3}-r_{1}\right)^{2} \ldots \ldots \ldots \ldots \ldots
\end{aligned}
$$

In the same manner as above-mentioned, $(d f / d r)_{r=r_{1}},\left(d^{2} f / d r^{2}\right)_{r_{=} r_{1}}$ are known; therefore, $K_{m}$ can be figured out.

Figure 2 given below shows the test piping which consists of a main pipe having $43 \mathrm{~mm}$

\begin{tabular}{|c|c|c|c|c|c|c|c|c|c|c|c|}
\hline \multirow{2}{*}{$\begin{array}{l}\text { Condi- } \\
\text { tion }\end{array}$} & \multirow{2}{*}{$Q_{5} \mathrm{~kg} / \mathrm{s}$} & \multicolumn{5}{|c|}{$\begin{array}{c}q_{m} / Q_{5} \\
m\end{array}$} & \multicolumn{5}{|c|}{$\begin{array}{c}k_{m} q_{m}^{2} \\
m\end{array}$} \\
\hline & & 1 & 2 & 3 & 4 & 5 & 1 & 2 & 3 & 4 & 5 \\
\hline 1 & $\begin{array}{l}5.235 \times 10^{-2} \\
5.550 \\
5.744 \\
5.972 \\
6.196\end{array}$ & $\begin{array}{l}0.115 \\
0.116 \\
0.115 \\
0.117 \\
0.117\end{array}$ & $\begin{array}{l}0.137 \\
0.137 \\
0.136 \\
0.139 \\
0.138\end{array}$ & $\begin{array}{l}0.171 \\
0.173 \\
0.174 \\
0.174 \\
0.174\end{array}$ & $\begin{array}{l}0.240 \\
0.239 \\
0.240 \\
0.238 \\
0.236\end{array}$ & $\begin{array}{l}0.337 \\
0.335 \\
0.334 \\
0.333 \\
0.336\end{array}$ & $\begin{array}{r}9.3 \\
10.5 \\
11.8 \\
12.4 \\
13.4\end{array}$ & $\begin{array}{l}13.0 \\
14.8 \\
15.5 \\
17.4 \\
18.5\end{array}$ & $\begin{array}{l}20.4 \\
23.4 \\
25.5 \\
27.3 \\
29.4\end{array}$ & $\begin{array}{l}40.2 \\
44.9 \\
48.4 \\
51.3 \\
54.3\end{array}$ & $\begin{array}{r}79.2 \\
88.0 \\
93.7 \\
100.7 \\
109.9\end{array}$ \\
\hline 2 & $\begin{array}{l}5.244 \times 10^{-2} \\
5.583 \\
5.854 \\
6.039 \\
6.452 \\
\end{array}$ & $\begin{array}{l}0.131 \\
0.132 \\
0.132 \\
0.133 \\
0.133 \\
\end{array}$ & $\begin{array}{l}0.156 \\
0.156 \\
0.155 \\
0.155 \\
0.156 \\
\end{array}$ & $\begin{array}{l}0.199 \\
0.198 \\
0.198 \\
0.199 \\
0.198 \\
\end{array}$ & $\begin{array}{l}0.271 \\
0.271 \\
0.271 \\
0.269 \\
0.270 \\
\end{array}$ & $\begin{array}{l}0.244 \\
0.243 \\
0.244 \\
0.244 \\
0.242 \\
\end{array}$ & $\begin{array}{l}11.9 \\
13.7 \\
15.2 \\
16.5 \\
18.8 \\
\end{array}$ & $\begin{array}{l}17.0 \\
19.4 \\
20.9 \\
22.2 \\
25.9 \\
\end{array}$ & $\begin{array}{l}27.6 \\
31.2 \\
34.2 \\
36.8 \\
41.5\end{array}$ & $\begin{array}{l}51.5 \\
58.1 \\
63.9 \\
67.2 \\
77.2 \\
\end{array}$ & $\begin{array}{r}99.1 \\
111.8 \\
123.9 \\
131.7 \\
148.5 \\
\end{array}$ \\
\hline \multirow[t]{2}{*}{$\begin{array}{l}\text { Condi- } \\
\text { tion }\end{array}$} & $Q_{5} \mathrm{~kg} / \mathrm{s}$ & \multicolumn{5}{|c|}{$\underset{m}{\left(K_{m}\right) Q_{5}^{2}}$} & \multicolumn{5}{|c|}{$\left(K_{m}\right) / \frac{1}{2 \mathrm{~g} \gamma}\left(\frac{1}{F}\right)^{2}$} \\
\hline & & 1 & 2 & 3 & 4 & 5 & 1 & 2 & 3 & 4 & 5 \\
\hline 1 & $\begin{array}{l}5.235 \times 10^{-2} \\
5.550 \\
5.744 \\
5.972 \\
6.196\end{array}$ & $\begin{array}{l}124.2 \\
139.5 \\
148.2 \\
159.6 \\
171.6\end{array}$ & $\begin{array}{l}120.5 \\
135.2 \\
144.5 \\
1546 \\
166.5\end{array}$ & $\begin{array}{l}113.1 \\
126.6 \\
134.5 \\
144.7 \\
155.6\end{array}$ & $\begin{array}{r}93.3 \\
105.1 \\
111.6 \\
120.7 \\
130.7\end{array}$ & $\begin{array}{l}54.3 \\
62.0 \\
66.3 \\
71.3 \\
75.1\end{array}$ & $\begin{array}{l}2.355 \\
2.353 \\
2.333 \\
2.325 \\
2.323\end{array}$ & $\begin{array}{l}2.284 \\
2.281 \\
2.275 \\
2.253 \\
2.254\end{array}$ & $\begin{array}{l}2.144 \\
2.136 \\
2118 \\
2.108 \\
2.106\end{array}$ & $\begin{array}{l}1.769 \\
1.773 \\
1.757 \\
1.759 \\
1.769\end{array}$ & $\begin{array}{l}1.029 \\
1.046 \\
1.044 \\
1.039 \\
1.017\end{array}$ \\
\hline 2 & $\begin{array}{l}5.244 \times 10^{-2} \\
5.583 \\
5.854 \\
6.039 \\
6.452\end{array}$ & $\begin{array}{l}130.6 \\
148.8 \\
159.8 \\
169.0 \\
193.2 \\
\end{array}$ & $\begin{array}{l}125.5 \\
143.1 \\
154.1 \\
163.1 \\
186.1\end{array}$ & $\begin{array}{l}114.9 \\
131.3 \\
140.8 \\
148.7 \\
170.5\end{array}$ & $\begin{array}{r}91.0 \\
104.4 \\
111.1 \\
118.3 \\
134.8\end{array}$ & $\begin{array}{l}43.4 \\
50.7 \\
51.1 \\
53.8 \\
63.5\end{array}$ & $\begin{array}{l}2.467 \\
2.433 \\
2.423 \\
2.408 \\
2.412\end{array}$ & $\begin{array}{l}2.371 \\
2.340 \\
2.337 \\
2.326 \\
2.323\end{array}$ & $\begin{array}{l}2.171 \\
2.147 \\
2.135 \\
2.118 \\
2.128\end{array}$ & $\begin{array}{l}1.719 \\
1.707 \\
1.685 \\
1.685 \\
1.683\end{array}$ & $\begin{array}{l}0.820 \\
0.829 \\
0.775 \\
0.766 \\
0.793\end{array}$ \\
\hline
\end{tabular}
diameter and a branch pipe $25 \mathrm{~mm}$ in diameter and $508 \mathrm{~mm}$ long with suction hood and 1007 mm pitch.

This pipe is suction type system as a whole and connects with $30 \mathrm{H}$. vacuum pump. There is a measuring nozzle between the pipe and the vacuum pump. A controlling resistance orifice can be inserted in the flange in the middle of each branch pipe. The flow of each branch pipe is figured out from the pressure difference at a distance of $120 \mathrm{~mm}$ from the top of the branch pipe. The results of this experiment are shown in Tables 1 and 2. The results of calculation by this method of adjust-

Table 1 
Table 2

\begin{tabular}{|c|c|c|c|c|c|c|c|c|c|c|}
\hline \multirow{2}{*}{$\begin{array}{l}\text { Condi. } \\
\text { tion }\end{array}$} & \multicolumn{5}{|c|}{$\left(K_{m b}\right) / \frac{1}{2 g \gamma}\left(\frac{1}{F}\right)^{2}$} & \multicolumn{5}{|c|}{$\begin{array}{c}q_{m} / Q_{5} \\
m\end{array}$} \\
\hline & 1 & 2 & 3 & 4 & 5 & 1 & 2 & 3 & 4 & 5 \\
\hline 1 & 2.338 & 2.269 & 2.122 & $1.7 \overline{65}$ & 1.035 & 0.116 & 0.137 & 0.173 & 0.239 & $0 . \overline{335}$ \\
\hline 2 & 2.429 & 2.339 & 2.140 & 1.696 & 0.797 & 0.132 & 0.156 & 0.198 & 0.270 & 0.243 \\
\hline
\end{tabular}

Table 3

\begin{tabular}{cccccc}
\hline $\begin{array}{l}\text { No. of } \\
\text { branch } \\
\text { pipe }\end{array}$ & $\left(K_{m}\right) r-\frac{1}{5} / \frac{1}{2 g \gamma}\left(\frac{1}{\mathrm{~F}}\right)^{2}$ & $\zeta_{m}$ & \multicolumn{3}{c}{ Diameter of orifice $d \mathrm{~mm}$} \\
\hline 1 & 2.816 & 0 & Calculation & Theorctical & Experimental \\
2 & 2.501 & 0.899 & - & - & -1.2 \\
3 & 2.141 & 1.926 & 19.5 & 20.1 & 21.10 \\
4 & 1.852 & 2.750 & 18.6 & 18.2 & 19.27 \\
5 & 0.686 & 6.076 & 16.5 & 16.6 & 16.65 \\
\hline
\end{tabular}

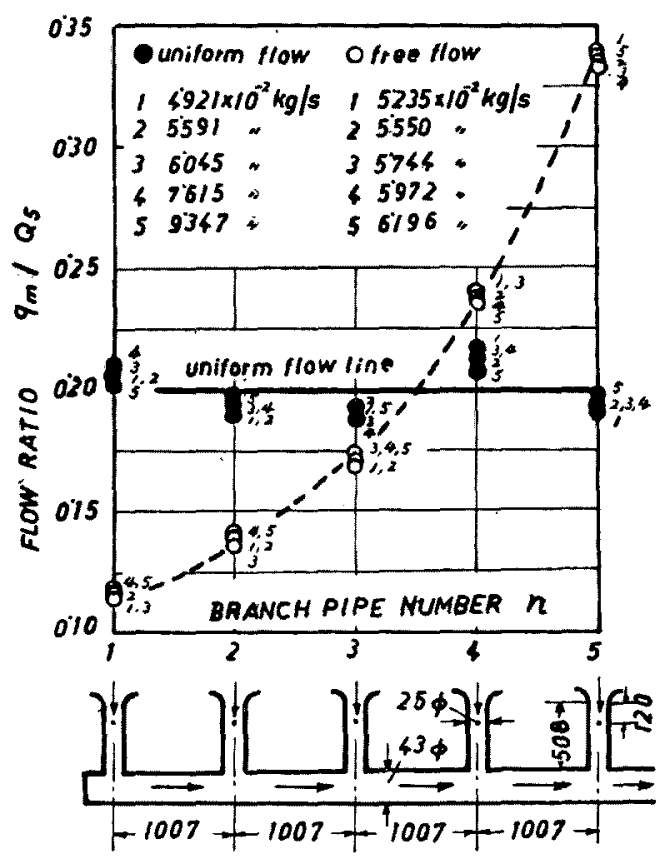

Fig. 2

ment (Method No. 2) are shown in Table 3. This table also includes values theoretically[6] obtainable.

\section{Flow Equation of Duct System with Parallel Blowers}

In the typical duct system as shown in Figure 3 , we have the following equations:

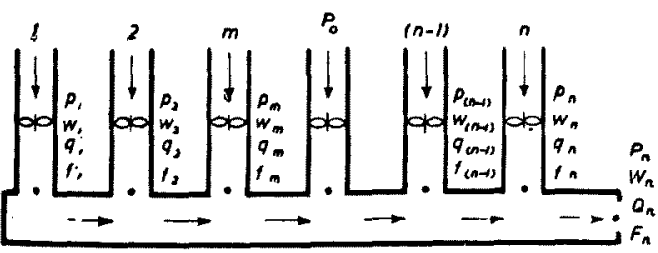

Fig. 3

$$
\begin{aligned}
P_{n}+\frac{\gamma}{2 g} w_{n}^{2}= & P_{n}+\zeta_{\min } \frac{\gamma}{2 g} W_{n}^{o}+\lambda \frac{L_{n}}{D_{n}} \frac{\gamma}{2 g} W_{n}^{o} \\
& +\frac{\gamma}{2 g} W_{n}^{2} \ldots \ldots \ldots \ldots \ldots \ldots \ldots \ldots \ldots \ldots \ldots
\end{aligned}
$$

$$
\begin{aligned}
& P_{n}+\frac{\gamma}{2 g} W_{n}^{2}=P_{0}+\zeta_{0} \frac{\gamma}{2 g} W_{n}^{2}+\frac{\gamma}{2 g} W_{n}^{2} \ldots \\
& Q_{n}=F_{n} W_{n} \gamma \quad \therefore W_{n}=\frac{Q_{n}}{F_{n} \gamma} \\
& q_{n}=f_{n} w_{n} \gamma \quad \therefore \ldots \ldots
\end{aligned}
$$

By the above relation we have

$$
\begin{aligned}
P_{n}-P_{0}= & \left(\zeta_{n n}+\lambda \frac{L_{n}}{D_{n}}+\zeta_{0}+1\right) \frac{1}{2 g \gamma} \\
& \left(\frac{1}{F_{n}}\right)^{2} Q_{n}^{2}-\frac{1}{2 g \gamma}\left(\frac{1}{f_{n}}\right)^{2} q_{n}^{2}
\end{aligned}
$$

Assuming $P_{n}-P_{0}$ make $\left(P_{n}\right)$, we have:

$$
\left(P_{n}\right)=K_{n} Q_{n}^{2}-k_{n} q_{n}^{2}
$$

where

$$
\begin{aligned}
& K_{n}=\left(\zeta_{n n}+\lambda \frac{L_{n}}{D_{n}}+\zeta_{0}+1\right) \frac{1}{2 g \gamma}\left(\frac{1}{F_{n}}\right)^{2} \\
& k_{n}=\frac{1}{2 g \gamma}\left(\frac{1}{f_{n}}\right)^{2}
\end{aligned}
$$

In the same manner, for the flow of branch $(n-1)$ we have

$$
\begin{aligned}
P_{(n-1)} & +\frac{\gamma}{2 g} w_{n}^{2}=P_{n}+\zeta_{b(n-1)} \frac{\gamma}{2 g} W_{(n-1)}^{2} \\
+ & \lambda \frac{L_{(n-1)}}{D_{(n-1)}} \frac{\gamma}{2 g} W_{(n-1)}^{2}+\zeta_{d n} \frac{\gamma}{2 g} W_{n}^{a} \\
+ & \lambda \frac{L_{n}}{D_{n}} \frac{\gamma}{2 g} W_{n}^{2}+\frac{\gamma}{2 g} W_{n}^{2} \ldots \ldots \ldots \ldots(40) \\
P_{(n-1)}-P_{0}= & \left(\zeta_{(n n-1)}+\lambda \frac{L_{(n-1)}}{D_{(n-1)}}\right) \frac{1}{2 g \gamma}\left(\frac{1}{F_{(n-1)}}\right)^{2} Q_{(n-1)}^{2} \\
& +\left(\zeta_{d n}+\lambda \frac{L_{n}}{D_{n}}+\zeta_{0}+1\right) \frac{1}{2 g \gamma}\left(\frac{1}{F_{n}}\right)^{2} Q_{n}^{2} \\
& -\frac{1}{2 g \gamma}\left(\frac{1}{f_{(n-1)}}\right)^{2} q_{n}^{2} \\
\left(P_{(n-1)}\right)= & K_{(n-1)} Q_{(n-1)}^{2}+K_{n}^{\prime} Q_{n}^{2}-k_{(n-1)} q_{(n-1)}^{2}
\end{aligned}
$$

where 


$$
\left\{\begin{array}{l}
K_{(n-1)}=\left(\zeta_{l(n-1)}+\lambda \frac{L_{(n-1)}}{D_{(n-1)}}\right) \frac{1}{2 g \gamma}\left(\frac{1}{F_{(n-1)}}\right)^{2} \\
K_{n}^{\prime}=\left(\zeta_{d n}+\lambda \frac{L_{n}}{D_{n}}+\zeta_{0}+1\right) \frac{1}{2 g \gamma}\left(\frac{1}{F_{n}}\right)^{2} \\
k_{(n-1)}=\frac{1}{2 g \gamma}\left(\frac{1}{f_{(n-1)}}\right)^{2}
\end{array}\right.
$$

Therefore, generally

$$
\left(P_{m}\right)=\sum_{m+1}^{n} K_{m}^{\prime} Q_{m}^{2}+K_{m} Q_{m}^{2}-k_{m} q_{m}^{2}
$$

where

$$
\left\{\begin{array}{l}
K_{m}=\left(\zeta_{b m}+\lambda \frac{L_{m}}{D_{m}}\right) \frac{1}{2 g \gamma}\left(\frac{1}{F_{m}}\right)^{2} \\
K_{m}^{\prime}=\left(\zeta_{d n}+\lambda-\frac{L_{m}}{D_{m}}\right) \frac{1}{2 g \gamma}\left(\frac{1}{F_{m}}\right)^{2} \\
k_{m}=\frac{1}{2 g \gamma}\left(\frac{1}{f_{m}}\right)^{2}
\end{array}\right.
$$

By using the following relation in the manner described above, general flow equations may be simplified as follows:

$$
\begin{aligned}
& \stackrel{n}{\stackrel{n}{n}} K_{m+1}^{\prime} Q_{m}^{2}+K_{m} Q_{m}^{2}=\left(K_{m}\right) Q_{n}^{2} \\
& \therefore\left(P_{n}\right)=\left(K_{n}\right) Q_{n}^{2}-k_{n} q_{n}^{2} \\
& \left(P_{(n-1)}\right)=\left(K_{(n-1)}\right) Q_{n}^{2}-k_{(n-1)} q_{(n-1)}^{2} \cdots \cdots \\
& \left(P_{m}\right)=\left(K_{m}\right) Q_{n}^{2}-k_{m} q_{m}^{2} \\
& \left(P_{2}\right)=\left(K_{2}\right) Q_{n}^{2}-k_{2} q_{2}^{2} \\
& \left(P_{1}\right)=\left(K_{1}\right) Q_{n}^{2}-k_{1} q_{1}^{2}
\end{aligned}
$$

\section{Uniform Distribution of Duct System with Parallel Blowers} then:

If each branch pipe keeps a uniform flow,

$$
q_{n}=q_{(n-1)}=\cdots=q_{m}=\cdots=q_{2}=q_{1} \equiv q
$$

Therefore, the flow ratio to the tctal quantity of flow is equal to $1 / n$, that is, $r=q / Q=1 / n$.

$$
\begin{aligned}
& \left(P_{n}\right)_{r=\frac{1}{n}}=\left(K_{n}\right)_{r-\frac{1}{n}} Q_{n}^{2}-k_{n} q^{2} \\
& \left(P_{\langle n-1\rangle}\right)_{r=\frac{1}{n}}=\left(K_{(n-1)}\right)_{r=\frac{1}{n}} Q_{n}^{2}-k_{(n-1)} q^{2} \cdots \\
& \left(P_{n}\right)_{r-\frac{1}{n}}=\left(K_{n}\right)_{r-\frac{1}{n}} Q_{n}^{2}-k_{n} q^{2} \ldots \ldots \ldots \ldots \\
& \left(P_{2}\right)_{r=\frac{1}{n}}=\left(K_{2}\right)_{r=\frac{1}{n}} Q_{n}^{2}-k_{2} q^{2} \\
& \left(P_{1}\right)_{r=\frac{1}{n}}=\left(K_{1}\right)_{r=\frac{1}{n}} Q_{n}^{2}-k_{1} q^{2}
\end{aligned}
$$

Now by Taylor expansion up to the second order, $\left(K_{n}\right)$ will be about as follws:

$$
\begin{gathered}
\left(K_{m}\right)_{r=\frac{1}{n}}=f\left(r_{1}\right)+\left(\frac{d f}{d r}\right)_{r=r_{1}}\left(\frac{1}{n}-r_{1}\right) \\
+\frac{1}{2 !}\left(\frac{d^{2} f}{d r^{2}}\right)_{r=r_{1}}\left(\frac{1}{n}-r_{1}\right)^{2} \cdots \cdots \cdots
\end{gathered}
$$

For the condition of 2,3

$$
\begin{aligned}
\left(K_{m}\right)_{2} & =f\left(r_{1}\right)+\left(\frac{d f}{d r}\right)_{r_{=} r_{1}}\left(r_{2}-r_{1}\right) \\
& +\frac{1}{2 !}\left(\frac{d^{2} f}{d r^{2}}\right)_{r=r_{1}}\left(r_{2}-r_{1}\right)^{2} \\
\left(K_{m}\right)_{3} & =f\left(r_{1}\right)+\left(\frac{d f}{d r}\right)_{r=r_{1}}\left(r_{3}-r_{1}\right) \\
& +\frac{1}{2 !}\left(\frac{d^{2} f}{d r^{2}}\right)_{r=r_{1}}\left(r_{3}-r_{1}\right)^{2}
\end{aligned}
$$

Therefore, $(d f / d r)_{r_{-} r_{1}},\left(d^{2} f / d r^{2}\right)_{r_{-} r_{1}}$ are calculated from the value of $\left(K_{m}\right)_{3},\left(K_{m}\right)_{3}$, so we know the value of $\left(K_{m}\right)_{r=\frac{1}{n}}$.

$$
Q_{n}=n q \quad\left(\frac{Q_{n}}{q}\right)^{2}=n^{2} .
$$

Accordingly

$$
\begin{aligned}
& \left(P_{n}\right)_{r=\frac{1}{n}} \mid q^{n}=\left(K_{n}\right)_{r=\frac{1}{n}} n^{2}-k_{n} \\
& \left(P_{(n-1)}\right)_{r=\frac{1}{n}} \mid q^{2}=\left(K_{(n-1)}\right)_{r=\frac{1}{n}} n^{2}-k_{(n-1)} \\
& \left(P_{m}\right)_{r=\frac{1}{n}} / q^{2}=\left(K_{m}\right)_{r=\frac{1}{n}} n^{2}-k_{m} \\
& \left(P_{2}\right)_{r=\frac{1}{n}} \mid q^{2}=\left(K^{2}\right)_{r=\frac{1}{n}} n^{2}-k_{2} \\
& \left(P_{1}\right)_{r=\frac{1}{n}} \mid q^{2}=\left(K_{1}\right)_{r=\frac{1}{n}} n^{2}-k_{1}
\end{aligned}
$$

However, blower pressure and quantity of flow are related by the performance curve of the blower. Therefore, generally:

$$
\begin{aligned}
& \left(P_{m}\right)=f\left(q_{m}\right) \\
& \left(P_{m}\right)_{r=\frac{1}{n}} \mid q^{2}=f(q) / q^{2}=A_{m}
\end{aligned}
$$

When the branch pipe and blowers are the same in the duct system

$$
\begin{aligned}
& k_{1}=k_{2}=\cdots=k_{(n-1)}=k_{n} \equiv \equiv k \\
& \left.\begin{array}{rl}
f\left(q_{1}\right) & =f\left(q_{2}\right)=\cdots=f\left(q_{(n-1)}\right) \\
& =f\left(q_{n}\right) \equiv f(q) \\
A_{1}=A_{2} & =\cdots=A_{(n-1)}=A_{n} \equiv \equiv A
\end{array}\right\}
\end{aligned}
$$

Therefore,

$$
\begin{gathered}
\left(K_{n}\right)_{r=\frac{1}{n}}=\left(K_{(n-1)}\right)_{r=\frac{1}{n}}=\cdots \cdots \\
\quad=\left(K_{2}\right)_{r=\frac{1}{n}}=\left(K_{1}\right)_{r=\frac{1}{n}} \cdots
\end{gathered}
$$

In this case, blower pressure is the function of $\mathrm{q}, \mathrm{s}$; therefore:

$$
\begin{aligned}
& \left(P_{m}\right)=f\left(q_{m}, s_{m}\right) \quad \therefore\left(P_{m}\right)_{r=\frac{1}{n}}=f\left(q, s_{m}\right) \\
& f\left(q, s_{n}\right) / q^{2}=\left(K_{n}\right)_{r=\frac{1}{n}} n^{2}-k \\
& f\left(q, s_{(n-1)}\right) / q^{2}=\left(K_{(n-1)}\right)_{r=\frac{1}{n}} n^{2}-k \cdots \\
& f\left(q, s_{m}\right) / q^{2}=\left(K_{m}\right)_{r=\frac{1}{n}} n^{2}-k \cdots \cdots \cdots(51) \\
& f\left(q, s_{2}\right) / q^{2}=\left(K^{2}\right)_{r=\frac{1}{n}} n^{2}-k \\
& f\left(q, s_{1}\right) / q^{2}=\left(K_{1}\right)_{r=\frac{1}{n}} n^{2}-k
\end{aligned}
$$




$$
\begin{aligned}
\therefore & \frac{f\left(q, s_{n}\right)-f\left(q, s_{(n-1)}\right)}{q^{2}}=\left\{\left(K_{n}\right)_{r=\frac{1}{n}}-\left(K_{(n-1)}\right)_{r=\frac{1}{n}}\right\} n^{2} \\
& \frac{f\left(q, s_{(n-1)}\right)-f\left(q, s_{(n-2)}\right)}{q^{2}}=\left\{\left(K_{(n-1)}\right)_{r=\frac{1}{n}}-\left(K_{(n-2)}\right)_{r=\frac{1}{n}}\right\} n^{2} \ldots \ldots \\
& \frac{f\left(q, s_{i}\right)-f\left(p, s_{2}\right)}{q^{2}}=\left\{\left(K_{3}\right)_{r=\frac{1}{n}}-\left(K_{2}\right)_{r=\frac{1}{n}}\right\} n^{2} \\
& \frac{f\left(q, s_{2}\right)-f\left(q, s_{1}\right)}{q^{2}}=\left\{\left(K_{n}\right)_{r=\frac{1}{n}}-\left(K_{1}\right)_{r=\frac{1}{n}}\right\} n^{2}
\end{aligned}
$$

Now, $f(q, s)$ may be written approximately by Taylor expansion up to the second order and by keeping $q$ equal to constant at the condition of $s=s_{n}$.

$$
f(q, s)=f\left(q, s_{n}\right)+\left(\frac{d f}{d s}\right)_{s=s_{n}}\left(s-s_{n}\right)+\frac{1}{2 !}\left(\frac{d^{2} f}{d s^{2}}\right)_{s=s_{n}}\left(s-s_{n}\right)^{2}
$$

In the same way as above mentioned, for the condition of 2,3 we have

$$
\left\{\begin{array}{l}
f_{2}(q, s)=f\left(q, s_{n}\right)+\left(\frac{d f}{d s}\right)_{s=s_{n}}\left(s_{z}-s_{n}\right)+\frac{1}{2 !}\left(\frac{d^{2} f}{d s^{2}}\right)_{s=s_{n}}\left(s_{2}-s_{n}\right)^{2} \\
f_{3}(q, s)=f\left(q, s_{n}\right)+\left(\frac{d f}{d s}\right)_{s=s_{n}}\left(s_{3}-s_{n}\right)+\frac{1}{2 !}\left(\frac{d^{2} f}{d s^{2}}\right)_{s=s_{n}}\left(s_{i}-s_{n}\right)^{2}
\end{array}\right.
$$

By the above equation, $(d f / d s)_{s=s_{n}},\left(d^{2} f / d s^{2}\right)_{s=s_{n}}$ are evaluated, so we have the following equation for $s-s_{n}=\Delta s$

$$
\begin{aligned}
& \frac{\left(\frac{d f}{d s}\right)_{s=s_{n}} S_{S_{(n-1)}}+\frac{1}{2 !}\left(\frac{d^{2} f}{d s^{2}}\right)_{s=s_{n}}\left(d_{S_{(n-1)}}\right)^{2}}{q^{2}}=\left\{K_{(n-1) r_{-\frac{1}{n}}}-\left(K_{n}\right)_{r_{-1} \frac{1}{n}}\right\} n^{2} \\
& \frac{\left(\frac{d f}{d s}\right)_{s=s_{n}} \Delta_{S_{(n-2)}}+\frac{1}{2 !}\left(\frac{d^{2} f}{d s^{2}}\right)_{s=s_{n}}\left(\Delta_{S_{(n-2)}}\right)^{2}}{q^{2}}=\left\{\left(K_{(n-2)}\right)_{r=\frac{1}{n}}-\left(K_{(n-1)}\right)_{r-\frac{1}{n}}\right\} n^{2} \ldots \ldots \\
& \frac{\left(\frac{d f}{d s}\right)_{s=s_{n}} \Delta_{S_{2}}+\frac{1}{2 !}\left(\frac{d^{2} f}{d s^{2}}\right)_{s=s_{n}}\left(A_{S_{2}}\right)^{2}}{q^{2}}=\left\{\left(K_{2}\right)_{r-\frac{1}{n}}-\left(K^{3}\right)_{r=\frac{1}{n}}\right\} n^{2} \\
& \frac{\left(\frac{d f}{d s}\right)_{s=s_{n}} \Delta_{S_{1}}+\frac{1}{2 !}\left(\frac{d^{2} f}{d s^{2}}\right)_{s=s_{n}}\left(\Delta_{S_{1}}\right)^{2}}{q^{2}}=\left\{\left(K_{1}\right)_{r=\frac{1}{n}}-\left(K_{2}\right)_{r=\frac{1}{n i}}\right\} n^{2}
\end{aligned}
$$

By these relations, the rate of increase in the revolutions of a blower can be figured out. If the rate to which the revolutions of a blower must be increased is an excessive rate which will make the fan unusable, the alternative is to use two fans in series and increase their revolutions to a safe maximum rate. The combined increased revolutions of the two blowers will equal the excessive rate but will not make the fans unusable.

\section{Conclusions}

(1) Our experiments have shown that the general flow equation, by both the pressure and suction duct systems, is the same in form. This discovery has led to the discovery of Method No. 1 by which the unknown factors can be controlled in order to obtain a uniform distribution.

(2) Method No. 2 is more practical and con- venient. It is based on the principle that blower or fan pressure $P$ is equal to the sum of equivalent pressure term of branch flow $q$ and equal to the sum of equivalent pressure term of main flow $Q$ nearest the blower or fan.

(3) Drag control method is not so effective as duct system of single blower.

(4) The effective method of flow control is to distribute the flow to each branch pipe by adjusting the revolutions of blowers so as to balance them with the duct pressure equation.

\section{Data Consulted}

(1) Authors: Report of Japan Cotton Technical Institute. Vol. 17, p. 6, (1954)

[2] Authors: Report of Japan Cotton Technical Institute, Vol. 25 , p. 3, (1954)

[3] Authors: Report of Japan Cotton Technical Institute, Vol. 26, p. 6, (1954)

[4] Authors: Report of Japan Cotton Technical Institute, Vol. 27. p. 3, (1954)

[5] I. Oki: J.S.M.E., Vol. 4, No. 14, p 77

[6] Authors: J.T.M.S., Vol. 5, No. 3, p 29, (1952) 